\title{
A influência das tecnologias na saúde mental dos idosos em tempos de pandemia: uma revisão integrativa
}

The Influence of Technologies on the mental health of the elderly in times of pandemic: na integrative review

La influencia de las tecnologías en la salud mental de las personas mayores en tiempos de pandemia: una revisión integradora

Debora Ellen Sousa Costa

ORCID: https://orcid.org/0000-0003-4205-8801

Universidade Federal do Maranhão, Brasil

E-mail: deborasousacosta@outlook.com

Sandryelle de Andrade Rodrigues

ORCID: https://orcid.org/0000-0002-0878-3885

Centro Universitário Doutor Leão Sampaio, Brasil

E-mail: sandryellerodrigues24@gmail.com

Rita de Cássia Loiola Alves

ORCID: https://orcid.org/0000-0002-0241-3939

Universidade Federal do Piauí, Brasil

E-mail: kassia.loiola@gmail.com

Milena Roberta Freire da Silva

ORCID: https://orcid.org/0000-0003-0203-4506

Universidade Federal de Pernambuco, Brasil E-mail: milena.freire@ufpe.br

Antônio Diego Costa Bezerra

ORCID: https://orcid.org/0000-0002-2441-2961

Centro Universitário Unifanor, Brasil Fundação Oswaldo Cruz, Brasil

E-mail: diegocostamjc@gmail.com

Daniel Coutinho dos Santos

ORCID: https://orcid.org/0000-0002-6230-9842

Universidade Federal do Maranhão, Brasil

E-mail: daniel.coutinho@discente.ufma.br

Milena Cordeiro de Freitas

ORCID: https://orcid.org/0000-0003-0208-9400 Faculdade Cearense, Brasil

E-mail: barra.milenafreitas@hotmail.com

Paula Ermans de Oliveira

ORCID: https://orcid.org/0000-0002-2735-5202 Universidade Potiguar, Brasil

E-mail: paulaaermans@gmail.com

Sabrina Freitas Nunes

ORCID: https://orcid.org/0000-0003-1442-0092

Universidade Regional do Cariri, Brasil

E-mail: sabrina2016acop@gmail.com

Victoria Caroline da Silva

ORCID: https://orcid.org/0000-0002-7091-6749

Centro Universitário Católica de Quixadá, Brasil

E-mail: vicctoriacsilva@outlook.com

Cidianna Emanuelly Melo do Nascimento

ORCID: https://orcid.org/0000-0001-5477-4413

Universidade Estadual do Ceará, Brasil

E-mail: profa.cidianna.melo@gmail.com

\section{Resumo}

Introdução: A COVID-19 configura-se como uma pandemia e atinge principalmente pessoas idosas, nesse sentido foram determinadas medidas de isolamento social para evitar a propagação do vírus. Para o estreitamento desse isolamento os idosos passaram a se familiarizar com as tecnologias. Objetivo: Abordar por meio de uma revisão integrativa da literatura como as tecnologias influenciam na saúde mental dos idosos em tempos de pandemia. Metodologia: Trata-se de uma revisão integrativa da literatura, onde foram realizadas buscas nas bases eletrônicas 
Scopus, Lilacs, Medline, Pubmed e Scielo, utilizando os descritores de acordo com o DeSC: Tecnologias em Saúde, Saúde Mental, Pandemia e Idoso, publicados entre 2019 e 2020. Para compor os resultados foram selecionados 9 artigos de acordo com os critérios de inclusão e exclusão previamente estabelecidos. Resultados e discussão: Observou-se que a maioria dos estudos $(77,77 \%)$ era da língua inglesa e $(44,44 \%)$ foram selecionados da Pubmed. Os autores abordaram que a inclusão digital de idosos e a utilização das tecnologias auxiliam na melhoria da saúde mental deste grupo; reduzem efeitos como a solidão e a sensação de isolamento social e possibilitam uma maior interação dos idosos com os amigos e familiares. Assim, essa ferramenta fortalece o sentimento de autonomia destes indivíduos e auxilia na redução da exclusão digital e do estigma de preconceito com a idade. Conclusão: Portanto, o uso das tecnologias por idosos proporcionam efeitos benéficos sobre a saúde mental desses, reduzindo sentimentos de solidão e a sensação de isolamento, além de proporcionar o aumento da sensação de segurança e independência.

Palavras-chave: COVID-19; Idoso; Isolamento social; Pandemia; Saúde mental.

\begin{abstract}
Introduction: The COVID-19 is configured as a pandemic and affects mainly elderly people. In this sense, social isolation measures were taken to prevent the spread of the virus. In order to reduce this isolation, the elderly started to become familiar with the technologies. Objective: To approach, through an integrative literature review, how technologies influence the mental health of the elderly in times of pandemic. Methodology: This is an integrative literature review, where searches were carried out on the electronic databases Scopus, Lilacs, Medline, Pubmed and Scielo, using the descriptors according to DeSC: Health Technology, Mental Health, Pandemia and Aged, published between 2019 and 2020. To compose the results, 9 articles were selected according to the inclusion and exclusion criteria previously established. Results and discussion: It was observed that most studies (77,77\%) were English language and $(44,44 \%)$ were selected from Pubmed. The authors addressed that the digital inclusion of the elderly and the use of technologies help to improve the mental health of this group; reduce effects such as loneliness and the feeling of social isolation and enable greater interaction between the elderly and friends and Family. Thus, this tool strengthens the feeling of autonomy of these individuals and helps to reduce the digital divide and the stigma of prejudice with age. Conclusion: Therefore, the use of technologies by the elderly provides beneficial effects on their mental health, reducing feelings of loneliness and the feeling of isolation, in addition to providing an increased sense of security and independence.
\end{abstract}

Keywords: COVID-19; Aged; Social isolation; Pandemic; Mental health.

\title{
Resumen
}

Introducción: COVID-19 se configura como una pandemia y afecta principalmente a personas mayores, en este sentido se tomaron medidas de aislamiento social para prevenir la propagación del virus. Para fortalecer este aislamiento, las personas mayores comenzaron a familiarizarse con las tecnologías. Objetivo: Abordar, a través de una revisión integradora de la literatura, cómo las tecnologías influyen en la salud mental de las personas mayores en tiempos de pandemia. Metodología: Se trata de una revisión integradora de la literatura, donde se realizaron búsquedas en las bases de datos electrónicas Scopus, Lilacs, Medline, Pubmed y Scielo, utilizando los descriptores según DeSC: Tecnologías Sanitarias, Salud Mental, Pandemia y anciano, publicados entre 2019 y 2020 . Para componer los resultados se seleccionaron 9 artículos de acuerdo con los criterios de inclusión y exclusión previamente establecidos. Resultados y discusión: Se observó que la mayoría de los estudios $(77,77 \%)$ fueron en inglés y $(44,44 \%)$ fueron seleccionados de Pubmed. Los autores abordaron que la inclusión digital de las personas mayores y el uso de tecnologías ayudan a mejorar la salud mental de este colectivo; reducen efectos como la soledad y el sentimiento de aislamiento social y permiten una mayor interacción entre los ancianos y los amigos y la familia. Así, esta herramienta fortalece el sentimiento de autonomía de estas personas y ayuda a reducir la brecha digital y el estigma del prejuicio con la edad. Conclusión: Por tanto, el uso de tecnologías por parte de las personas mayores proporciona efectos beneficiosos sobre su salud mental, reduciendo la sensación de soledad y la sensación de aislamiento, además de proporcionar una mayor sensación de seguridad e independencia.

Palabras clave: COVID-19; Anciano; Aislamiento social; Pandemia; Salud mental.

\section{Introdução}

As pandemias são conhecidas como epidemias que se espalham rapidamente por diversos países e afetam uma quantidade relativamente grande de pessoas, gerando consequências de diversas naturezas. De acordo com a Organização Mundial da Saúde (OMS), o novo coronavírus, nomeado SARS-CoV-2 (Síndrome Respiratória Aguda Grave Coronavírus 2) foi anunciada ao mundo em 31 de dezembro de 2019, e em março de 2020 já era declarado como Emergência de Saúde Pública de Importância Internacional (Duarte et al. 2020; Silva et al. 2020).

Devido à escassez de tratamentos que demonstrem eficácia para a COVID- 19, a OMS recomenda o contato limitado com o intuito de reduzir a propagação do vírus e, o consequente aumento dos casos (Ahmeda et al. 2020; Martínez et al. 2020). 
É demonstrado que a população idosa se encontra entre as mais acometidas por essa patologia, requerendo uma atenção mais especializada a fim de minimizar os efeitos desastrosos que essa pode causar (Hammerschmidt \& Santana, 2020).

Dados apontam que a COVID-19 apresenta maior taxa de mortalidade entre pessoas com 80 anos ou mais, principalmente em indivíduos com doenças crônicas. A pandemia aflorou o destaque aos idosos, principalmente devido ao potencial risco dessa população. Nesse sentido, foram direcionadas estratégias de distanciamento social especificamente para esse grupo, assim como, medidas que visassem o estreitamento do distanciamento social por meio da utilização de ferramentas tecnológicas (Hammerschmidt \& Santana, 2020).

Um dos grandes diferenciais evidenciados por esta pandemia em relação às anteriores, trata-se do nível de globalização da sociedade, uma vez que, essa encontra-se mais conectada com o ambiente digital (Coelho et al. 2020). A utilização de tecnologias da informação e comunicação auxilia no enfrentamento de diversos desafios, uma vez que os idosos podem utilizar essa ferramenta para otimizar os atendimentos voltados para saúde, permitindo ao utente obter maior autonomia, além de evitar o deslocamento, assim como pode proporcionar um maior contato social mesmo que de forma virtual, principalmente para aqueles que vivem isolados ou acompanhados por outros idosos (Marlene et al. 2020).

Mesmo os idosos apresentando uma menor familiaridade com a tecnologia, essa permite minimizar as limitações consequentes do isolamento, como por exemplo chamadas por vídeo ou pedido de comida e remédios via aplicativos, além de atuar diretamente na saúde, como subsídio para promoção do bem-estar e da qualidade de vida, pois ajudam, por exemplo, a reduzir a depressão e até mesmo a sensação de solidão, pois o meio virtual permite um maior contato dos idosos com os familiares e amigos (Nabuco et al. 2020).

Diante desta realidade, é notório que a utilização desses recursos tecnológicos também é importante na divulgação de conteúdos educacionais, pois a pandemia tem imposto diversas restrições e novas configurações sociais para os idosos, sendo essa uma ferramenta que auxilia a população na aquisição de conhecimento acerca da pandemia, além de possibilitar uma maior interatividade com diversas realidades (Camacho et al. 2020).

Desta forma, o presente estudo tem como objetivo abordar por meio de uma revisão integrativa da literatura como as tecnologias influenciam na saúde mental dos idosos em tempos de pandemia.

\section{Metodologia}

Trata-se de um estudo bibliográfico, do tipo revisão integrativa, que proporciona o aprofundamento do tema estudado, pois engloba e sintetiza resultados de pesquisas sobre uma determinada temática, de maneira organizada e sistemática, seguindo seis etapas: definição da pergunta norteadora ou questão de pesquisa; busca ou amostragem na literatura baseados nos critérios de inclusão e exclusão do estudo; extração de informações dos trabalhos coletados; avaliação minuciosa e crítica dos estudos incluídos; sintetização e interpretação dos resultados; apresentação da revisão integrativa com um produto (Galvão, 2008; Souza et al. 2010).

Para o direcionamento do estudo formulou-se a seguinte pergunta norteadora: "Como são abordadas na literatura as tecnologias voltadas à saúde mental dos idosos na pandemia? ". Os critérios de inclusão utilizados foram: trabalhos na forma de texto completo; com assunto compatível sobre a temática de tecnologias aplicadas à saúde mental na pandemia; indexados nas bases de dados em português, inglês ou espanhol; com disponibilidade gratuita na íntegra e publicados entre 2019 e 2020. Os critérios de exclusão foram: publicações duplicadas, relatos de experiências, editoriais, dissertações e teses.

A busca do material bibliográfico foi realizada nos meses de outubro e novembro de 2020 nas bases de dados: Scopus, Lilacs, Medline, Pubmed e Scielo. Para realizar a busca, utilizou-se, a partir da consulta no DECS e no MESH, a junção dos descritores: Tecnologias em Saúde (Health Technology), Saúde Mental (Mental Health), Pandemia (Pandemic) e Idoso (Aged), usando o operador booleano "and". 
Como resultado da busca nas bases de dados escolhidas, foram encontrados 8.101 artigos, utilizando os descritores e os critérios de inclusão estabelecidos. Após a leitura do título e resumo selecionaram-se 18 artigos. Posteriormente, feito a leitura do artigo completo e retirando os duplicados, selecionaram-se ao final 9 artigos. O fluxograma de seleção dos artigos pode ser identificado na Figura 1.

Como parte da avaliação crítica, foi realizada uma leitura minuciosa dos estudos em sua totalidade e a releitura dos resultados, com o intuito de verificar e retirar os pontos marcantes e em destaque, para agrupamento das categorias. A análise temática foi conduzida por 2 autoras e revisada por 3 autoras.

Os estudos foram avaliados quanto aos níveis de evidência de acordo com a proposta estabelecida por Melnyk \& Fineout-Overholt (2011), são organizados por um sistema de classificação que divide os estudos em sete níveis: nível I evidências provenientes de revisões sistemáticas, meta análises, ensaios clínicos randomizados controlados; nível II evidências obtidas de ensaios clínicos randomizados bem delineado; nível III - evidências originadas de ensaios clínicos bem delineados sem randomização; nível IV - evidências derivadas de estudos do tipo caso controle e coorte; nível V-evidências procedentes de revisões sistemáticas de estudos descritivos e qualitativos; nível VI - evidências retiradas de um único estudo qualitativo ou descritivo; e nível VII - evidências propostas pela opinião de autoridades e/ou relatórios emitidos por comitês de especialistas.

Figura 1. Fluxograma de seleção dos artigos sobre o uso das tecnologias relacionadas à saúde mental pelos idosos na pandemia. 


\section{Resultados e Discussão}

Nesta revisão foram selecionados 9 artigos, indexados entre os anos de 2019 e 2020, sendo um (11,11\%) de 2019 e oito $(88,88 \%)$ de 2020 . Dentre os artigos selecionados, sete $(77,77 \%)$ encontravam-se em revistas em língua inglesa e dois $(22,22 \%)$ em língua portuguesa, dos quais um (11,11\%) foi identificado na Lilacs, um $(11,11 \%)$ na Medline, dois $(33,33 \%)$ na Scopus, quatro $(44,44 \%)$ na Pubmed e um $(11,11 \%)$ na Scielo. Dos 9 artigos, quatro $(44,44 \%)$ estavam localizados em jornais e periódicos interdisciplinares em saúde pública, três $(33,33 \%)$ em revistas, jornais e anais em outras áreas da saúde (enfermagem, psicologia e medicina) e dois $(22,22 \%)$ em revistas e jornais no ramo da geriatria. Foi elaborado um quadrosíntese, explicitando as principais informações contidas em cada artigo, tais como ano, autor, tipo de estudo, local de estudo e os principais achados (Tabela 1$)$.

Tabela 1. Descrição dos estudos que abordaram a influência das tecnologias na saúde mental dos idosos na pandemia.

\begin{tabular}{|c|c|c|c|c|}
\hline Autor/Ano & Objetivo & $\begin{array}{c}\text { Tipo de Estudo / } \\
\text { Nível de } \\
\text { Evidência }\end{array}$ & $\begin{array}{c}\text { Local de } \\
\text { Estudo }\end{array}$ & Principais Achados \\
\hline $\begin{array}{l}\text { TAVARES, D. M. } \\
\text { S. et al. }(2020) \text {. }\end{array}$ & $\begin{array}{l}\text { Descrever a média total de } \\
\text { independência geral e por } \\
\text { domínios de comunicação } \\
\text { dos idosos da comunidade } \\
\text { e verificar as variáveis } \\
\text { sociodemográficas e de } \\
\text { saúde associadas à maior } \\
\text { média de independência de } \\
\text { comunicação entre os } \\
\text { idosos. }\end{array}$ & $\begin{array}{c}\text { Estudo } \\
\text { quantitativo } \\
\mathrm{V}\end{array}$ & Brasil & $\begin{array}{l}\text { O estudo demonstrou que grande parte dos } \\
\text { idosos }(6,88) \text { utiliza o processo de } \\
\text { comunicação relacionado as necessidades } \\
\text { básicas e }(6,79) \text { voltado para a comunicação } \\
\text { social. Essas médias de independência de } \\
\text { comunicação foram associadas aos idosos } \\
\text { que apresentavam escolaridade. }\end{array}$ \\
\hline $\begin{array}{l}\text { TSERTSIDIS, A.; } \\
\text { KOLKOWSKA, E.; } \\
\text { HEDSTRÖM, K. } \\
\text { (2019). }\end{array}$ & $\begin{array}{l}\text { Identificar os fatores que } \\
\text { influenciam a aceitação da } \\
\text { tecnologia para o } \\
\text { envelhecimento em vigor } \\
\text { por idosos na fase de pós- } \\
\text { implementação. }\end{array}$ & $\begin{array}{l}\text { Revisão de } \\
\text { literatura } \\
\text { VI }\end{array}$ & Suécia & $\begin{array}{l}\text { Diante da revisão realizada os autores } \\
\text { demonstraram que o processo de aceitação } \\
\text { da tecnologia na fase de pós-implementação } \\
\text { para os idosos é influenciada por diversos } \\
\text { fatores, sendo esses categorizados em: } \\
\text { preocupações/problemas relacionados à } \\
\text { tecnologia, características positivas da } \\
\text { tecnologia utilizada, benefícios que a } \\
\text { tecnologia pode oferecer, necessidade de } \\
\text { tecnologia e influência social. A literatura } \\
\text { mostrou que as tecnologias digitais que } \\
\text { apoiam o envelhecimento em fase de pós- } \\
\text { implementação ainda têm baixa adesão. }\end{array}$ \\
\hline $\begin{array}{l}\text { MENEZES J. A. et } \\
\text { al. (2020). }\end{array}$ & $\begin{array}{l}\text { Problematizar uma das } \\
\text { dimensões do projeto que } \\
\text { é a articulação entre } \\
\text { contexto-afeto-texto, } \\
\text { abordando um dos temas } \\
\text { presentes nas histórias } \\
\text { videogravadas } \\
\text { veiculadas no Instagram } \\
\text { que é a relação entre morte } \\
\text { e memória. }\end{array}$ & $\begin{array}{l}\text { Pesquisa- } \\
\text { intervenção, } \\
\text { modalidade de } \\
\text { investigação } \\
\text { qualitativa } \\
\text { VI }\end{array}$ & Brasil & $\begin{array}{l}\text { O estudo demonstrou que a utilização de } \\
\text { tecnologias tais como celulares, } \\
\text { computadores, redes sociais, aplicativos de } \\
\text { reuniões e encontros virtuais mantem as } \\
\text { pessoas que estão isoladas em conexão. }\end{array}$ \\
\hline
\end{tabular}




\begin{tabular}{|c|c|c|c|c|}
\hline $\begin{array}{l}\text { VAN TILBURG, T. } \\
\text { G. et al. (2020). }\end{array}$ & $\begin{array}{l}\text { Analisar se a redução } \\
\text { consequente na frequência } \\
\text { de contatos sociais, perdas } \\
\text { pessoais e a experiência de } \\
\text { ameaças gerais na } \\
\text { sociedade ocasionaram } \\
\text { redução do bem-estar. }\end{array}$ & $\begin{array}{c}\text { Estudo } \\
\text { observacional } \\
\text { VI }\end{array}$ & Holanda & $\begin{array}{l}\text { A solidão social e emocional e a saúde } \\
\text { mental de } 1.679 \text { participantes - com idades } \\
\text { entre } 65-102 \text { anos -, foram medidas no } \\
\text { início de outubro e novembro de } 2019 \text { e em } \\
\text { maio de } 2020 \text {. Os dados da pesquisa } \\
\text { demonstraram que a solidão dos idosos } \\
\text { aumentou nesse período. Esse fator, } \\
\text { juntamente a perdas pessoais, preocupações } \\
\text { com a pandemia e um declínio na confiança } \\
\text { nas instituições sociais, contribuíram para o } \\
\text { aumento dos problemas de saúde mental } \\
\text { nesse público. }\end{array}$ \\
\hline $\begin{array}{c}\text { GUSTAVSSON, J.; } \\
\text { BECKMAN, L. } \\
\text { (2020). }\end{array}$ & $\begin{array}{l}\text { Analisar as consequências } \\
\text { das recomendações de } \\
\text { distanciamento físico e } \\
\text { isolamento social para a } \\
\text { saúde mental dos idosos na } \\
\text { Suécia durante a fase } \\
\text { inicial da pandemia do } \\
\text { COVID -19. }\end{array}$ & $\begin{array}{c}\text { Estudo } \\
\text { observacional } \\
\text { analítico } \\
\text { VI }\end{array}$ & Suécia & $\begin{array}{l}\text { Os resultados mostraram que cerca de } \\
\text { metade dos participantes relatou que a } \\
\text { pandemia COVID-19 contribuiu para } \\
\text { problemas de saúde mental em termos de, } \\
\text { por exemplo, problemas de sono e sintomas } \\
\text { de depressão. O suporte social percebido } \\
\text { por meio de tecnologias de comunicação } \\
\text { contemporâneas pode neutralizar e diminuir } \\
\text { a sensação de isolamento. }\end{array}$ \\
\hline $\begin{array}{l}\text { LEE, K.; JEONG, } \\
\text { G.; YIM, J. (2020). }\end{array}$ & $\begin{array}{l}\text { Analisar e identificar } \\
\text { fatores que possam estar } \\
\text { relacionados a saúde } \\
\text { psicológica e mental do } \\
\text { idoso durante o COVID-19 } \\
\text { através de uma revisão } \\
\text { teórica. }\end{array}$ & $\begin{array}{c}\text { Estudo } \\
\text { bibliográfico } \\
\text { VI }\end{array}$ & Coreia & $\begin{array}{l}\text { Idosos com relações familiares } \\
\text { estremecidas e baixa solidariedade se } \\
\text { sentem mais deprimidos e solitários. Assim, } \\
\text { o apoio e as relações familiares podem ser a } \\
\text { solução para a proteção da saúde } \\
\text { psicológica e mental dos idosos em situação } \\
\text { de pandemia. O contato com a família e } \\
\text { amigos por meio da mídia social, chamadas } \\
\text { telefônicas e internet, por exemplo, bem } \\
\text { como manutenção das atividades religiosas } \\
\text { de forma online, podem contribuir para } \\
\text { envolvimento social e superação de crises } \\
\text { pessoais. }\end{array}$ \\
\hline $\begin{array}{l}\text { LEKAMWASAM, } \\
\text { R.; } \\
\text { LEKAMWASAM, } \\
\text { S. (2020). }\end{array}$ & $\begin{array}{l}\text { Analisar os efeitos da } \\
\text { pandemia de COVID-19 } \\
\text { na saúde e no bem-estar de } \\
\text { pessoas idosas por meio de } \\
\text { uma revisão de literatura. }\end{array}$ & $\begin{array}{c}\text { Estudo } \\
\text { bibliográfico } \\
\text { VI }\end{array}$ & Sri Lanka & $\begin{array}{l}\text { O distanciamento social e o isolamento } \\
\text { podem desencadear, em pessoas mais } \\
\text { velhas, morbidades psicológicas, como } \\
\text { depressão e má qualidade do sono. Muitas } \\
\text { vezes, esse público apresenta dificuldades } \\
\text { no manejo das tecnologias, que poderiam } \\
\text { mitigar o isolamento e seus efeitos } \\
\text { psicológicos. Dessa forma, faz-se } \\
\text { necessário que medidas sejam tomadas para } \\
\text { inclusão dos idosos no âmbito tecnológico. }\end{array}$ \\
\hline $\begin{array}{l}\text { ROLANDI, E. et al. } \\
\qquad(2020)\end{array}$ & $\begin{array}{l}\text { Explorar como idosos, } \\
\text { previamente treinados para } \\
\text { o uso de Sites de Redes } \\
\text { Sociais } \\
\text { vivenciaram o período de } \\
\text { bloqueio. }\end{array}$ & $\begin{array}{c}\text { Estudo de coorte } \\
\text { IV }\end{array}$ & Itália & $\begin{array}{l}\text { Os participantes treinados para o uso de } \\
\text { SNSs relataram um uso significativamente } \\
\text { maior de SNSs e redução da sensação de } \\
\text { serem deixados de fora. Em comparação } \\
\text { com os níveis pré-bloqueio, os indivíduos } \\
\text { treinados para o uso de SNSs mostraram } \\
\text { uma redução mais leve nos contatos sociais. } \\
\text { Esses achados apoiam a utilidade do } \\
\text { treinamento de idosos para o uso de SNSs, } \\
\text { a fim de melhorar sua inclusão social, } \\
\text { mesmo em condições extremas de auto- } \\
\text { isolamento e vulnerabilidade percebida. }\end{array}$ \\
\hline
\end{tabular}




\begin{tabular}{|c|c|c|c|c|}
\hline $\begin{array}{l}\text { SUN, X. et al. } \\
\quad(2020)\end{array}$ & $\begin{array}{l}\text { Examinar o estado atual do } \\
\text { uso da Internet por idosos } \\
\text { e os fatores que o } \\
\text { influenciam (incluindo } \\
\text { físicos, psicológicos e } \\
\text { sociais), bem como a } \\
\text { demanda entre os idosos } \\
\text { por serviços inteligentes. }\end{array}$ & $\begin{array}{c}\text { Estudo } \\
\text { transversal } \\
\text { VI }\end{array}$ & China & $\begin{array}{l}\text { O estudo demonstrou que, dos } \\
\text { participantes, } 38,6 \% \text { utilizavam a Internet. } \\
\text { A atividade online favorita deles foi o } \\
\text { namoro online }(74,2 \%) \text {, e as informações } \\
\text { de saúde que obtiveram pela Internet } \\
\text { estavam principalmente relacionadas à dieta } \\
(63,1 \%) \text { e exercícios }(47,1 \%) \text {. Os sujeitos } \\
\text { demandaram pulseiras inteligentes (MD = } \\
2,80) \text { e chamadores de emergência (MD = } \\
\text { 2,77). Gênero, idade, escolaridade, renda } \\
\text { mensal, qualidade de vida, número de } \\
\text { amigos e participação social influenciam no } \\
\text { uso da Internet. }\end{array}$ \\
\hline
\end{tabular}

Fonte: Autores.

A análise dos estudos na finalidade de identificar como as tecnologias voltadas à saúde mental dos idosos na pandemia estão sendo abordadas na literatura, possibilitou a construção de duas categorias: I- inclusão digital para a melhoria da saúde mental de idosos em isolamento social e II- motivações e barreiras para a utilização das tecnologias pelos idosos.

\section{Categoria I - A inclusão digital para a melhoria da saúde mental de idosos em isolamento social}

Os estudos incluídos nesta categoria evidenciam a importância da inclusão digital de idosos e a utilização das tecnologias para a melhoria da saúde mental deste grupo de indivíduos (Figura 2). Dentre os estudos, dois demonstram que durante o processo de isolamento social, as tecnologias têm o papel de reduzir efeitos adversos à saúde mental de idosos (Gustavsson \& Beckman, 2020; Sun et al. 2020), tais como a solidão (Sun et al. 2020) e a sensação de isolamento (Gustavsson \& Beckman, 2020).

Além disso, cinco artigos descrevem benefícios provenientes do uso da internet e das tecnologias no bem-estar dos idosos, tais como a manutenção do contato social (Tsertsidis et al. 2019; Sun et al. 2020; Van Tilburg et al. 2020), e essa interação social do idoso, possibilita um envelhecimento ativo (Tavares et al. 2020); o aumento da sensação de segurança e proteção, da independência e do prazer (Tsertsidis et al. 2019), além de possibilitar um bem-estar psicológico e melhorar a satisfação com a vida (Sun et al. 2020), mantendo os isolados conectados (Menezes et al. 2020).

Ademais, um dos estudos também aborda estratégias para o uso de tecnologias em benefício da saúde mental dos idosos. Entre as estratégias estão: manter contato com familiares por meio de mídia social e internet e participar de atividades religiosas de forma online (Lee et al. 2020). Por conseguinte, um artigo de revisão enfatiza medidas para incluir os idosos no meio digital, tais como: programas regulares e motivacionais para ajudar os idosos a ultrapassarem este período de pandemia; recebimento de pacotes gratuitos ou descontos para falarem com familiares e amigos; apresentação de aplicativos e de métodos de comunicação (Lekamwasam \& Lekamwasam, 2020). 
Figura 2. Importância da inclusão digital para a melhoria da saúde mental de idosos em isolamento social.

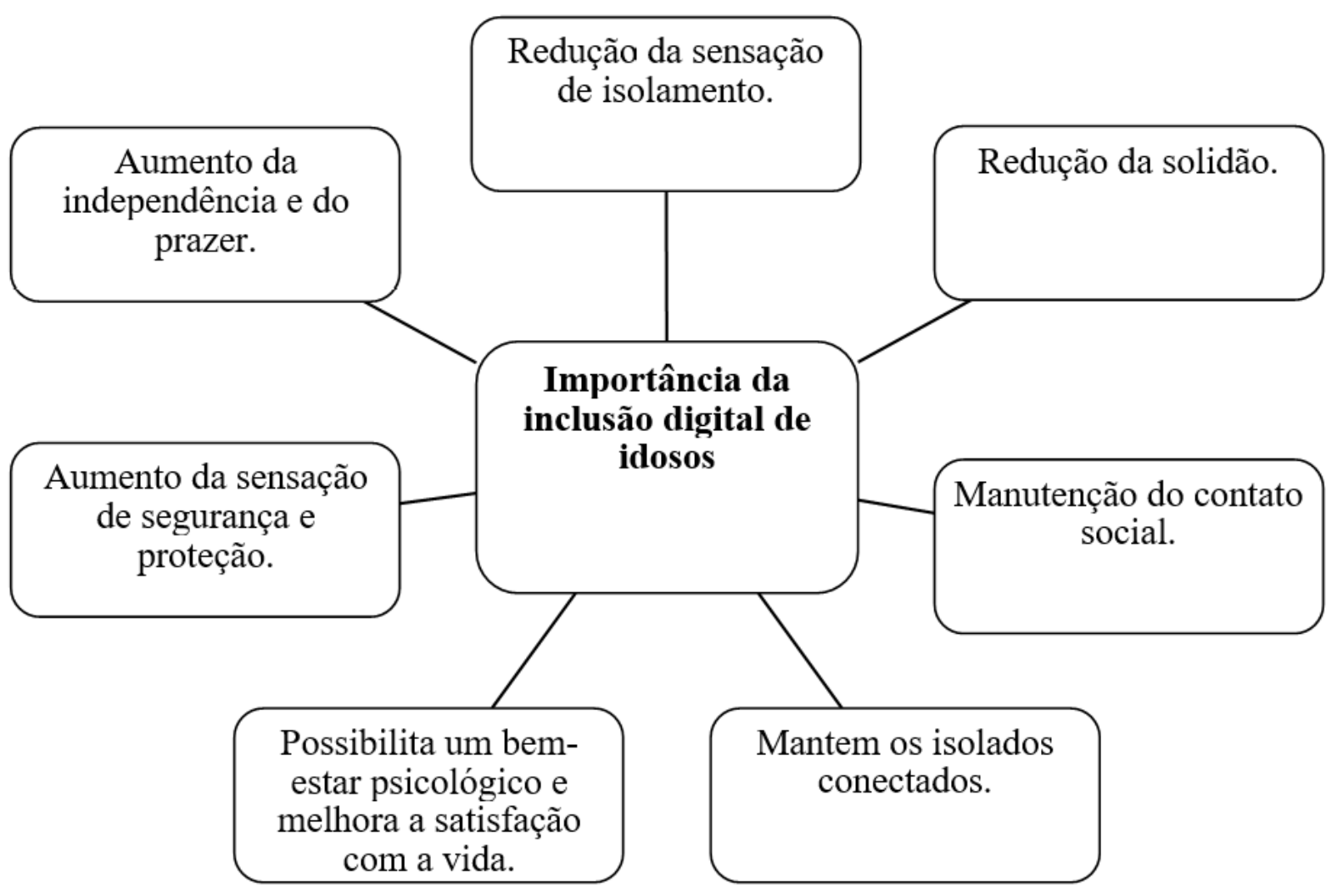

Fonte: Autores.

Com o advento da pandemia a necessidade de comunicação, a preocupação com amigos e familiares, promoveu novas atitudes em busca de informações, nesta ótica, uma das alternativas encontradas foi a utilização da tecnologia digital, empregada para estreitar o distanciamento social, principalmente entre os idosos (Velho \& Herédia, 2020). A inclusão digital dos idosos e o uso da Internet proporciona melhora do contato social e familiar, além destes manifestarem satisfação com o aprendizado e com as oportunidades que a inclusão digital proporciona nas atividades diárias, no entretenimento, na preservação da saúde mental, na motivação para aprender o novo, na melhoria da memória e no rompimento de obstáculos que são impostos pela sociedade (Casadei et al. 2019). Idosos que têm acesso ao ensino digital apresentam melhor performance cognitiva, linguística e redução dos sintomas de depressão (Yassuda \& Cachioni, 2019), onde é demonstrado que a tecnologia exerce efeito positivo sobre a saúde mental do idosos (Gustavsson \& Beckman, 2020; Sun et al. 2020).

O grupo de extensão "Movimentando o Corpo e a Mente na Terceira Idade" da Universidade Federal do Rio Grande do Norte (UFRN) durante a pandemia promoveu a criação de um grupo de WhatsApp, visando fomentar a informação em saúde, principalmente sobre a Covid-19 para idosos. Durante o desenvolvimento das atividades foi notório que o grupo proporcionou uma maior interação entre esses indivíduos, assim como o fortalecimento de laços de amizade. No que concerne, esse também oportunizou além da interação social, a prevenção de doenças que podem ser causadas pelo isolamento (ansiedade, depressão, etc), uma vez que, os idosos comentavam seus medos e aflições do atual cenário, as dificuldades enfrentadas pelo isolamento, atividades diárias e momentos de diversão (Oliveira et al. 2020).

Durante o isolamento social é importante preservar a comunicação constante com familiares e amigos, por ligações de telefones e via redes sociais, como meio de buscar apoio e reduzir o tédio e sensação de solidão (Brooks et al. 2020; Lee et al. 2020). Como estratégia para facilitar a utilização dos meios digitais, evidenciou que aplicativos de celular disponíveis na 
PlayStore que tivessem como característica a facilidade de uso e atendesse as necessidades do idoso restrito à sua residência, seria uma alternativa para melhorar a independência e a redução de danos durante o isolamento (Banskota et al. 2020).

É válido salientar que de acordo com diversos autores, a inclusão digital para idosos exerce diversos benefícios positivos sobre a saúde dos mesmos em tempos de pandemia, no entanto, apesar dessa ser um mecanismo positivo, ainda é necessário instaurar medidas de vigilância aos idosos, para que se possa ter um controle da mídia, pois utilizada de forma errônea pode acarretar em pânico, medo e insegurança. Todavia, as consequências decorrentes desse uso inadequado a longo prazo ainda são desconhecidas (Holmes et al. 2020).

\section{Categoria II- Motivações e barreiras para a utilização de tecnologias pelos idosos}

A segunda categoria contempla os fatores de motivação e barreira para o uso de tecnologias abordados nos artigos pelos idosos participantes dos estudos (Figura 3).

Figura 3. Motivações e barreiras para a utilização das tecnologias pelos idosos identificados nos estudos submetidos à análise da temática.

\begin{tabular}{|c|}
\hline Motivações \\
\hline Entretenimento \\
\hline Comunicação \\
\hline $\begin{array}{c}\text { Interação social e } \\
\text { interpessoal }\end{array}$ \\
\hline Acesso à informação \\
\hline $\begin{array}{c}\text { Redução da sensação de } \\
\text { isolamento }\end{array}$ \\
\hline Promoção da \\
independência \\
\hline Privacidade \\
\hline Descrição \\
\hline
\end{tabular}

Barreiras

Funcionamento técnico

Dificuldade no manuseio

Custo dos aparelhos

Designer inadequado

Limitação física e funcional

Insegurança

Não confiabilidade

Fonte: Autores.

Fatores como a promoção do entretenimento, relacionado ao ato de ouvir música ou ver vídeos; aumento da comunicação e da interação interpessoal e social, voltadas para o uso das redes sociais como Facebook e Whatsapp, e para as conversas estabelecidas com parentes e conhecidos; além do acesso às informações, tanto relacionadas à procura por conteúdos de saúde ou notícias; redução da sensação de isolamento e promoção de uma vida independente, a respeito da possibilidade de manutenção da própria saúde e dos sentimentos de bem-estar, foram descritos pelos estudos italiano e chinês (Sun et al. 2020; Rolandi et al. 2020). 
Além disso, a manutenção da privacidade e a descrição no momento do uso das tecnologias foram relatados como pontos motivadores por idosos, nos resultados de uma revisão de literatura realizada por suecos (Tsertsidis et al. 2019). Ademais, também foram verificados os pilares da motivação dos idosos frente a utilização das tecnologias, onde, segundo o estudo chinês e a revisão de literatura sueca, a necessidade e o tempo de uso das tecnologias; a qualidade de vida e a influência social por parte da família, amigos e organizações, atuam como base dos pontos motivadores (Sun et al. 2020; Tsertsidis et al. 2019).

Em relação às barreiras, participantes idosos dos estudos realizados na China, Itália e a revisão de literatura realizada por suecos, relataram que as questões técnicas de funcionamento; dificuldades no gerenciamento e manuseio da tecnologia; o custo dos aparelhos com relação direta sobre a renda; design inadequado e divergente da preferência do idoso; limitações físicas e funcionais, como visão reduzida e deficiência física; além do sentimento de pouca confiabilidade e segurança, se caracterizaram como limitadores da incorporação das tecnologias pelos idosos (Sun et al. 2020; Rolandi et al. 2020; Tsertsidis et al. 2019).

A aproximação do meio familiar por meio da tecnologia, bem como o incentivo e a participação de atividades com amigos e familiares, aumenta significativamente a motivação dos idosos. A interação social e a manutenção de contatos possibilitam que os idosos entendam as tecnologias digitais como ferramentas úteis e necessárias, possíveis de serem utilizadas por eles. Essa percepção desperta o sentimento de autonomia e auxilia na redução da exclusão digital e do estigma de preconceito de idade no uso de tecnologias, por meio do acesso a estas de forma intergeracional (Sun et al. 2020; Rolandi et al. 2020).

Outrossim, com o uso da tecnologia os idosos sentem-se mais independentes e responsáveis por si, possuindo maior consciência e controle sobre a sua condição de saúde, por terem acesso a informações sobre saúde online. Dessa forma, os idosos apresentam-se mais seguros e confiantes em suas casas, mesmo que estejam sozinhos. Todavia, embora muitos idosos sintam-se seguros, alguns ainda relatam pouca confiabilidade nos sistemas de segurança da internet, temendo por suas privacidades. (Sun et al. 2020; Tsertsidis et al. 2019).

A utilização das tecnologias digitais de saúde para serviços de telemedicina, chamadas de emergência e monitoramento de saúde móvel representam a maior parte do interesse dos idosos. Entretanto, o design e o manuseio de muitos dispositivos eletrônicos são vistos como complexos e limitantes, dificultando o suprimento das demandas de gerenciamento básico de saúde e serviços médicos para os idosos de forma online, reduzindo assim a parcela desses que demonstra aptidão, motivação e condições necessárias para uso dos mesmos (Sun et al. 2020; Rolandi et al. 2020; Tsertsidis et al. 2019).

Não é incomum deparar-se com idosos que apresentem limitações físicas e funcionais, sofram de visão reduzida e/ou possuam dificuldades de memória. Esses fatores, juntamente ao fato da internet e da tecnologia terem tomado maior proporção quando eles já apresentavam meia-idade ou idade avançada, atrapalham o processo de aprendizagem e manuseio dos dispositivos tecnológicos. Por esse motivo, grande parte dos idosos relata ter aprendido a utilizar a internet por meio dos familiares e jovens, realidade prejudicada quando não se possui vínculos familiares e/ou sociais bem estabelecidos e presentes no cotidiano (Sun et al. 2020; Rolandi et al. 2020).

A situação de renda é considerada uma limitação para muitos idosos, tendo em vista que a falta de apoio financeiro suficiente pode afetar diretamente o poder de compra e o acesso deste público aos dispositivos tecnológicos. A chamada “corrida tecnológica” resulta em produtos cada vez mais sofisticados e inovadores, mas nem sempre acessíveis. Além disso, os efeitos da pandemia do COVID - 19 ocasionaram impactos na economia, tendo como consequência o aumento do preço de produtos eletrônicos, distanciando ainda mais de uma grande parcela dos idosos o uso tecnologia digital (Sun et al. 2020; Tsertsidis et al. 2019). 


\section{Conclusão}

Diante das dificuldades enfrentadas pelos idosos em manter a saúde mental durante o período de isolamento social, oriundo de ações preventivas à propagação do novo Coronavírus SARS-CoV-2, demonstrou-se o papel das tecnologias na saúde mental destes indivíduos em isolamento, com a importância no que tange a redução da solidão e da sensação de isolamento, o aumento das sensações de segurança, proteção, independência e do prazer, melhorando o estado psicológico, a satisfação com a vida e o contato social. Desta forma, tornou-se evidente a necessidade de medidas de inclusão dos idosos no meio digital.

Ademais, pôde-se concluir que a utilização das tecnologias pelos idosos possuía fatores motivadores relacionados ao entretenimento, comunicação e interação interpessoal/social, acesso à informação e promoção de uma vida independente, sendo que estes fatores poderiam sofrer influência das relações sociais e familiares; necessidade e tempo de uso das tecnologias e pela qualidade de vida. Por outro lado, aspectos como questões técnicas de funcionamento; dificuldades na utilização das tecnologias; o custo dos aparelhos; limitações físicas e funcionais e o sentimento de pouca confiabilidade e segurança, representam barreiras para essa incorporação das tecnologias por parte dos idosos.

\section{Referências}

Ahmed, M. Z., Ahmed, O., Aibao, Z., Hanbin, S., Siyu, L., \& Ahmad, A. (2020). Epidemic of COVID-19 in China and associated Psychological Problems. Asian journal of psychiatry, 102092.

Alvarenga, G. M. de O., Yassuda, M. S., \& Cachioni, M. (2019). Inclusão digital com tablets entre idosos: metodologia e impacto cognitivo. Psicologia, Saúde \& Doenças, 20(2), 384-401.

Banskota, S., Healy, M., \& Goldberg, E. M. (2020) 15 Smartphone Apps for Older Adults to Use While in Isolation During the COVID-19 Pandemic. Western Journal of Emergency Medicine, 21(3), 514.

Brooks, S. K., Webster, R. K., Smith, L. E., Woodland, L., Wessely, S., Greenberg, N., \& Rubin, G. J. (2020). The psychological impact of quarantine and how to reduce it: rapid review of the evidence. The Lancet.

Camacho, A. C. L. F., Silva, R. T., \& Souza, V. M. F. (2020). Tecnologia da informação ao idoso em tempos de COVID-19. Research, Society and Development, 9(6), e124963497-e124963497.

Casadei, G. R., Bennemann, R. M., \& Rodrigues, T. F. (2019). Influência das redes sociais virtuais na saúde dos idosos. Enciclopédia Biosfera, 16 (29), 1962.

Coelho, A. L., Morais, I. de A., \& Rosa, W. V. S. (2020). A utilização de tecnologias da informação em saúde para o enfrentamento da pandemia do Covid-19 no Brasil. Cadernos Ibero-Americanos de Direito Sanitário, 9(3), 183-199.

Duarte, M. de Q., Santo, M. A. S., Lima, C. P., Giordani, J. P., \& Trentini, C. M. (2020). COVID-19 e os impactos na saúde mental: uma amostra do Rio Grande do Sul, Brasil. Ciência \& Saúde Coletiva, 25, 3401-3411.

Galvão, C. M. (2008). Revisão integrativa: método de pesquisa para a incorporação de evidências na saúde e na enfermagem. Texto \& Contexto Enferm.17(4), 758-64.

Gustavsson, J., \& Beckman, L. (2020). Compliance to Recommendations and Mental Health Consequences among Elderly in Sweden during the Initial Phase of the COVID-19 Pandemic-A Cross Sectional Online Survey. International Journal of Environmental Research and Public Health, 17(15), 5380.

Hammerschmidt, K. S. de A. \& Santana, R. F. Saúde do idoso em tempos de pandemia COVID-19. Cogitare Enfermagem, 25.

Holmes, E. A., O’Connor, R. C., Perry, V. H., Tracey, I., Wessely, S., Arseneault, L., Ballard, C., Christensen, H., Silver, R. C., Everall, I., Ford, T., John, A., Kabir, T., King, K., Madan, I., Michie, S., Przybylski, A. K., Shafran, R., Sweeney, A., Worthman, M., Yardley, L., Cowan, K., Cope, C., Hotopf, M., \& Bullmore, E. (2020). Multidisciplinary research priorities for the COVID-19 pandemic: a call for action for mental health science. The Lancet Psychiatry.

Lee, K., Jeong, G., \& Yim, J. (2020). Consideration of the Psychological and Mental Health of the Elderly during COVID-19: A Theoretical Review. International Journal of Environmental Research and Public Health, 17(21), 8098.

Lekamwasam, R., \& Lekamwasam, S. (2020). Effects of COVID-19 Pandemic on Health and Wellbeing of Older People: A Comprehensive Review. Annals of Geriatric Medicine and Research, 24(3), 166.

Marlene, R., Morouço, P., Carreira, I. F. R., Nogueira, A., Oliveira, A., Mendes, D., Ribeiro, D., Homem, F., Zambana, J., Pereira, L., Simões, S., Graur, M., Bairrada, C., Azul, J., Calado, M., Brito, S., Ferreira, F., Castro, R., Costa, R., Góis, S., Fonseca, E., Ferreira, L., Baltazar, N., Sousa, R., \& Alves, J. (2020). Tecnologia e inovação ao serviço do exercício e saúde. Exercício. Pandemia COVID-19. Tecnologia Vs. Isolamento Social.

Martínez, V. B., Kapczinski, F. P., Cardoso, T. de A., Atienza-Carbonell, B., Rosa, A. R., Mota, J. C. da., \& De Boni, R. B. (2020). The assessment of lifestyle changes during the COVID-19 pandemic using a multidimensional scale. Revista de Psiquiatría y Salud Mental. 
Research, Society and Development, v. 10, n. 2, e8210212198, 2021

(CC BY 4.0) | ISSN 2525-3409 | DOI: http://dx.doi.org/10.33448/rsd-v10i2.12198

Menezes, J. de A., Botelho, S. S., Da Silva, R. A., Santos, A. C. de H., Leão, D. S. de S., Canales, V. F., Da Silva, H. L., Da Silva, I. N. F., \& Dos Santos, B. V. (2020). A contação de histórias no instagram como tecnologia leve em tempos pesados de pandemia. Psicologia \& Sociedade, 32.

Nabuco, G., Oliveira, M. H. P. P. de., \& Afonso, M. P. D. (2020). O impacto da pandemia pela COVID-19 na saúde mental. Revista Brasileira de Medicina de Família e Comunidade, 15(42), 2532-2532.

Oliveira, J., Costa, D. G. da., Nascimento, D. D. do., Nunes, V. M. de A., \& Nobre, T. T. X. (2020). A construção de um envelhecimento ativo: estratégia virtual de promoção e cuidado com a saúde do idoso frente à pandemia do novo coronavírus.

Rolandi, E., Vaccaro, R., Abbondanza, S., Casanova, G., Pettinato, L., Colombo, M., \& Guaita, A. (2020). Loneliness and Social Engagement in Older Adults Based in Lombardy during the COVID-19 Lockdown: The Long-Term Effects of a Course on Social Networking Sites Use. International journal of environmental research and public health, 17(21), 7912.

Silva, A. F. da., Estrela, F. M., Lima, N. S., \& Abreu, C. T. de A. (2020). Saúde mental de docentes universitários em tempos de pandemia. Physis: Revista de Saúde Coletiva, 30, e300216.

Souza, M. T., Silva, M. D., \& Carvalho, R. (2010). Revisão integrativa: o que é e como fazer. Einstein, 8(1), 102-106.

Sun, X., Yan, W., Zhou, H., Wang, Z., Zhang, X., Huang, S., \& Li, L. (2020). Internet use and need for digital health technology among the elderly: a crosssectional survey in China. BMC public health, 20(1), 1-8.

Tavares, D. M. dos S., Oliveira, N. G. N., Marchiori, G. F., Santana, L. P. M., Guimarães, M. S. F., \& Jardim, J da C. (2020). Fatores associados à independência de comunicação entre idosos da comunidade. Revista Enfermagem UERJ, 28, 49370

Tsertsidis, A., Kolkowska, E., \& Hedström, K. (2019). Factors influencing seniors' acceptance of technology for ageing in place in the post-implementation stage: A literature review. International journal of medical informatics, 129, 324-333.

Van Tilburg, T. G., Steinmetz, S., Stolte, E., Van Der Roest, H., \& Vries, D. H. de. (2020). Loneliness and mental health during the COVID-19 pandemic: A study among Dutch older adults. The Journals of Gerontology: Series B.

Velho, F. D., \& Herédia, V. B. M. (2020). O Idoso em Quarentena e o Impacto da Tecnologia em sua Vida. Rosa dos Ventos, 12(3), 1-14. 